\title{
Effect of a concurrent training session with different intensities in post-exercise blood pressure responses in normotensive adults
}

\author{
André Luiz F Medeiros ${ }^{1}$, Manoela Vieira Sousa2* ${ }^{*}$, Morgana Lunardi², Silas Nery Oliveira², \\ Cintia de la Rocha Freitas ${ }^{2}$
}

\begin{abstract}
The purpose of this study was to analyse the effect of a concurrent training session (CT) with different intensities in post-exercise blood pressure responses in normotensive adults. Ten normotensive adult males (30.7 \pm 4.1 years) participated in this study, they performed four experimental protocols: a) Strength Training (ST) at $50 \%$ of $1 \mathrm{RM}$ and aerobic training (AT) between $50-60 \%$ of the Working Heart Rate (WHR); b) ST at $50 \%$ of 1RM and AT between $80-90 \%$ of the WHR; c) ST at $85 \%$ of 1 RM and AT between $50-60 \%$ of the WHR; d) ST at $85 \%$ of 1 RM and AT between $80-90 \%$ of the WHR. Every protocol presented a decrease in the systolic blood pressure (SBP) 1 minute after finishing the training session, however, only the protocol with the intensity of 50\% $1 \mathrm{RM}$ and $80-90 \%$ WHR presented a significant hypotensive effect $(\mathrm{p} \leq \mathrm{0.05})$. None of the protocols presented a significant decrease in the diastolic blood pressure. We concluded that the hypotensive effect in the SBP can be observed when the CT is performed at the intensity of $50 \%$ of 1 RM and of $80-90 \%$ of the WHR.
\end{abstract}

Keywords: Post-exercise hypotension; Strength Training; Aerobic Training; Concurrent Training; Heart Rate.

\section{INTRODUCTION}

Physical exercises have been used as strategies to reduce the arterial blood pressure (ABP) in hypertensive individuals (Gambassi et al., 2016). Acute physical exercise has shown decreased values in the post-exercise blood-pressure (BP) when compared to the pre-exercise resting values, this phenomenon is called post-exercise hypotension (PEH) (Barreto et al., 2015; Polito \& Farinatti, 2006). These post-exercise BP decreases have occurred mainly in hypertensive individuals (Polito \& Farinatti, 2006), which substantiates the medical recommendation of physical exercise as a non-pharmacological strategy to control and prevent arterial hypertension (AH) (Senna et al., 2016).

Mazzoccante et al. (2016) cite studies that observed a PEH both through strength training (ST) and aerobic training (AT). In this perspective the concurrent training (CT) emerges, which can be defined as the combined practice of aerobic and strength exercise in the same training session, being recently presented as an effective anti-hypertensive treatment (Corso et al., 2016). However, the PEH effect is not fully understood due to the training protocol variations regarding the types of exercise, duration, frequency and intensity (Gambassi et al., 2016). Therefore, the greater the understanding regarding the $\mathrm{PEH}$, the more effective the exercise tends to be in decreasing the BP.

Thus, it is necessary to evaluate how these training variables can be manipulated and what is their influence in healthy populations, such as young adults, to understand and adapt the methods and procedures for posterior use in specific populations (Vilaça-Alves et al., 2016). Therefore, the American College of Sports Medicine (ACSM) recommendations for healthy adult individuals were applied for ST and AT (Garber et al., 2011). In this sense, the purpose

\footnotetext{
Manuscript received at April $7^{\text {th }} 2017$; Accepted at June $8^{\text {th }} 2017$

${ }^{1}$ Federal University of Santa Catarina, Florianópolis, SC, Brazil.

${ }^{2}$ Laboratory of Biomechanics, Post-Graduation Program in Physical Education, Federal University of Santa Catarina, Florianópolis, SC, Brazil.

*Corresponding author: Biomechanics Laboratory, Sports Center, Federal University of Santa Catarina, University Campus - Neighborhood Trindade. Mailbox 476. Zip Code: 88040-900. Florianópolis, SC. Brazil. E-mail: manoelavsousa@gmail.com
} 
of this study was to analyse the effect of a CT session with different intensities in the postexercise arterial blood pressure in normotensive adults. We hypothesize that $\mathrm{CT}$ provides a reduction in post-exercise $\mathrm{BP}$ about pre-exercise $\mathrm{BP}$ and when prescribed at a higher intensity, CT exercises influence a more significant hypotension.

\section{METHOD}

\section{Participants}

Ten normotensive males participated in this study, all practitioners of ST for at least six months without interruption and at least three times a week. The criteria to be included in this study were: (a) the absence of any muscle or joint injury or any medical contraindications; (b) negative answers to all the questions in the Physical Activity Readiness Questionnaire (PARQ); (c) the non-consumption of any substance that could alter the cardiovascular responses of the participants. All the subjects were informed of the procedures before they signed the free and clarified consent term. The present study has the registered approval of the Human Research Ethics Committee of the Joana de Gusmão Children's Hospital, under the number 956.006.

\section{Instruments}

Physical evaluation and arterial blood pressure evaluation

Anthropometric measurements of height and body mass were taken according to the procedures established by Guedes and Guedes (2006), with a stadiometer and a Welmy scale (WELMY-110, São Paulo, Brazil) with accuracy of $0.5 \mathrm{~cm} \quad 0.1 \mathrm{~kg}$, respectively. The ABP measurements were taken with an automatic digital device Omron M3 Intellisense (HEM-7051E), validated under the European Society of Hypertension (EHS) International Protocol (Akpolat, Erdem, \& Aydogdu, 2012). All the BP reading procedures followed the recommendations established by the Brazilian Cardiology Society, being performed three readings, with 1-minute intervals between each one, on the left arm. The readings were taken before every training session, immediately after ending the session and every 10 minutes during the 60 minutes of post-exercise recuperation.

\section{Six-Rep Max test (6RM)}

All the subjects performed a test and retest session consisting of 6-maximal repetitions (6RM). The participants initiated the test with lighter loads and then progressed to heavier loads to determine the 6RM load in the following exercises: bench press, low row, $45^{\circ}$ inclination leg press and squats using a squat machine. All the subjects had three attempts with 5-minute intervals between each attempt to find the 6RM load and 20-minute breaks between the exercises and the tests were redone after 48 hours to establish reproducibility. To reduce the margin of error, the following procedures were adopted: (a) standardized instructions were supplied to the participants before the test; (b) the participant was instructed regarding the exercise execution technique during the different stages of the execution; (c) the evaluator was alert of the position adopted by the participant at the moment of the evaluation; and (d) the evaluator gave verbal encouragement to maintain an elevated stimulation level during the execution of the exercises.

\section{Procedures}

Training Program

The AT was prescribed using the working heart rate (WHR) obtained by multiplying the target intensity by percentage by the reserve heart rate and was added to the resting heart rate. The equation proposed by Tanaka, Monaham, and Seals (2001) was used to establish the maximum heart rate and for the resting heart rate was used the equation of Guedes and Guedes (2006). Regarding the ST, the 6RM test data were used to estimate the load of one-repetition maximum (1RM) with the aid of the formula suggested by Guedes and Guedes (2006). The participants randomly performed just one session of the four CT protocols: $\mathrm{P} 1$ (ST at $50 \%$ of $1 \mathrm{RM}$ and $\mathrm{AT}$ between $50-60 \%$ of the WHR), P2 (ST at $50 \%$ of 1RM and AT between $80-90 \%$ of the WHR), P3 (ST at $85 \%$ of $1 \mathrm{RM}$ and AT between $50-60 \%$ of the WHR), P4 (ST at $85 \%$ of $1 \mathrm{RM}$ and AT between $80-90 \%$ of the WHR). The intensity of 
aerobic training and strength was prescribed according to the ACSM recommendations on aerobic training intensity and strength areas, when performed individually (Garber et al., 2011). During the training session, the strength and aerobics exercises were alternated. For the ST, three sets of repetitions to concentric failure were performed, with 45 seconds of rest between the sets. For the AT, 5-minute sets were performed on a treadmill.

\section{Statistical Analysis}

In order to describe this study's variables, we used descriptive statistics. To verify the data normalization, the Shapiro-Wilk test was performed, and the analysis of the differences found in the pre and post SBP and diastolic blood pressure (DBP) values was performed through the analysis of variance for repeated measurements, followed by the Bonferroni post-hoc test. The data were analysed using the statistical package SPSS version 22.0 , considering a $5 \%(\mathrm{p} \leq$ 0.05) significance level.

\section{RESULTS}

Table 1 shows the group characteristics, considering the participants individual data. Participants presented mean age of $30.7 \pm 4.1$ years old, body mass index of $25.9 \pm 3.3 \mathrm{~kg} / \mathrm{m}^{2}$, RSBP of $118.1 \pm 8.3 \mathrm{mmHg}$, RDBP $67.8 \pm 6.0$ $\mathrm{mmHg}$ and RHR of $65.9 \pm 11.6 \mathrm{bpm}$.
Table 1.

Characterization of the variables (mean $\pm s d$ ) of the research subjects $(n=10)$.

\begin{tabular}{lc}
\hline \multicolumn{1}{c}{ Variables } & Average \pm SD \\
\hline Age (years) & $30.7 \pm 4.1$ \\
Height $(\mathrm{cm})$ & $174.7 \pm 3.9$ \\
Body Mass $(\mathrm{kg})$ & $79.0 \pm 11.8$ \\
IMC $\left(\mathrm{kg} / \mathrm{m}^{2}\right)$ & $25.9 \pm 3.3$ \\
RSBP $(\mathrm{mmHg})$ & $118.1 \pm 8.3$ \\
RDBP $(\mathrm{mmHg})$ & $67.8 \pm 6.0$ \\
RHR $(\mathrm{bpm})$ & $65.9 \pm 11.6$ \\
\hline
\end{tabular}

SD: standard deviation; RSBP: resting systolic blood pressure; RDBP: resting diastolic blood pressure, RHR: resting heart-rate.

The data presented in Table 2 show systolic and DBP before and after each exercise protocol. In SBP, significant differences were observed in $\mathrm{P} 2(137.1 \pm 11.5 \mathrm{mmHg})$ and $\mathrm{P} 4(136.9 \pm 16.2$ $\mathrm{mmHg}$ ) exercise protocols at the blood pressure values immediately after the end of the concurrent training session $(\mathrm{p}<0.05)$, when compared to the pre-exercise values (P2: $118.3 \pm$ $4.5 \mathrm{mmHg}$ and $\mathrm{P} 4: 116.4 \pm 8.0 \mathrm{mmHg}$ ). Significant differences in hypotension acute postexercise were observed in P2 exercise protocol at 30' (110.6 $\pm 6.3 \mathrm{mmHg})$ and 40' $(108.1 \pm 5.0$ $\mathrm{mmHg}$ ) when compared to pre-exercise values. In the DBP, significant increase in DBP was observed immediately after P4 compared to the pre-exercise value $(70.2 \pm 8.2 \mathrm{mmHg}$ vs. $62.9 \pm$ $7.2 \mathrm{mmHg}$, respectively).

Table 2.

Mean ( \pm sd) Systolic Blood Pressure values (SBP) and Diastolic Blood Pressure values (DBP), in mmHg, before (pre) and after the experimental session (from 1 to $60 \mathrm{~min}$ ).

\begin{tabular}{lcccccccc}
\hline & \multicolumn{2}{c}{ P1 } & \multicolumn{2}{c}{ P2 } & \multicolumn{2}{c}{ P3 } & \multicolumn{2}{c}{ P4 } \\
& SBP & DBP & SBP & DBP & SBP & DBP & SBP & DBP \\
\hline Pre & $117.4 \pm 8.4$ & $64.9 \pm 6.9$ & $118.3 \pm 4.5$ & $64 \pm 6.6$ & $122.4 \pm 9.9$ & $65.9 \pm 5.1$ & $116.4 \pm 8.0$ & $62.9 \pm 7.2$ \\
$\mathbf{1}^{\prime}$ & $126 \pm 14.1$ & $66.4 \pm 10.2$ & $137.1 \pm 11.5^{*}$ & $69 \pm 7.3$ & $131.3 \pm 12.7$ & $69.1 \pm 7.5$ & $136.9 \pm 16.2^{*}$ & $70.2 \pm 8.2^{*}$ \\
$\mathbf{1 0}$ & $116.5 \pm 9.2$ & $65.4 \pm 6.6$ & $114.7 \pm 5.0$ & $62.6 \pm 7.1$ & $116.8 \pm 9.9$ & $65 \pm 6.4$ & $115.5 \pm 9.1$ & $65.1 \pm 8.9$ \\
$\mathbf{2 0}^{\prime}$ & $114.1 \pm 9.4$ & $64.4 \pm 7.6$ & $114.2 \pm 6.3$ & $63.3 \pm 6.9$ & $115.5 \pm 9.9$ & $63.9 \pm 6.2$ & $110.4 \pm 8.5$ & $64.4 \pm 9.4$ \\
$\mathbf{3 0}$ & $113.2 \pm 11.4$ & $62.1 \pm 7.3$ & $110.6 \pm 6.3^{*}$ & $64.1 \pm 6.0$ & $112.5 \pm 7.4$ & $64.7 \pm 6.4$ & $111.4 \pm 7.6$ & $63.3 \pm 7.2$ \\
$\mathbf{4 0}$ & $111.2 \pm 11.5$ & $62.7 \pm 7.6$ & $108.1 \pm 5.0^{*}$ & $62.4 \pm 5.0$ & $112.6 \pm 8.7$ & $64.4 \pm 5.4$ & $113.8 \pm 7.2$ & $63.7 \pm 7.9$ \\
$\mathbf{5 0}$ & $110.4 \pm 11.0$ & $61.8 \pm 5.6$ & $111.3 \pm 6.2$ & $61.2 \pm 6.4$ & $112.7 \pm 9.6$ & $64.2 \pm 5.3$ & $108.6 \pm 6.8$ & $62.8 \pm 4.2$ \\
$\mathbf{6 0}$ & $113.2 \pm 8.4$ & $64.3 \pm 6.1$ & $111 \pm 6.8$ & $65 \pm 5.2$ & $111.7 \pm 9.3$ & $66 \pm 4.6$ & $108.1 \pm 7.8$ & $63.3 \pm 6.8$ \\
\hline
\end{tabular}

P1 (ST at $50 \%$ of 1RM and AT between $50-60 \%$ of the WHR), P2 (ST at 50\% of 1RM and AT between $80-90 \%$ of the WHR), P3 (ST at $85 \%$ of 1 RM and AT between $50-60 \%$ of the WHR), P4 (ST at $85 \%$ of 1 RM and AT between $80-90 \%$ of the WHR). * significant difference $\mathrm{p}<0.05$ comparing the pre-exercise moment with the post-exercise moment. 


\section{DISCUSSION}

The purpose of this study was to analyse the effect of a CT session with different intensities in the post exercise blood pressure response in normotensive adults. The main finding of the study was the reduction of the SBP caused by the CT performed at $50 \%$ of $1 \mathrm{RM}$ at the ST and between $80-90 \%$ of the WHR (P2). Concurrent Training with exercises at higher intensity was more effective in reducing SBP, however, the intensity of this training model did not influence the participants DBP variation, partially accepting our hypothesis.

The other protocols did not present a decrease in the SBP and the DBP, meaning they did not cause $\mathrm{PEH}$. The increase in the SBP observed immediately after the $\mathrm{P} 2$ training $(\mathrm{p}=0.002)$ and P4 $(\mathrm{p}=0.032)$ was observed in other studies (Vilaça-Alves et al., 2016; Saldanha et al., 2016) and can be explained by the increase in the chemoreceptors' activation due to peripheral fatigue (Mediano, Paravidino, Simão, Pontes, \& Polito, 2005).

When comparing the SBP between the pre and post exercise moments done with each protocol, there was a significant difference in minute 30 $(\mathrm{p}=0.05)$ and minute $40(\mathrm{p}<0.001)$ in P2 (80$90 \%$ of the WHR), presenting PEH. Aerobic Training applied on the ST can influence hypotensive responses (Cunha, Santos, Massaferri, Monteiro, \& Farinatti, 2013), which could explain the protocols of highest intensities in the AT reductions post exercise. Additionally, low-intensity ST favours a greater number of repetitions and a longer time of muscular tension, which contribute to a better cardiovascular response due to the greater afferent muscular nervous stimulation, mediated by mechanisms associated to the mechanorreflex and metaborreflex controls (Cunha et al., 2013), which may have contributed to the appearance of PEH after P2.

Santiago et al. (2013) compared the influence of the exercises order (AT followed by ST and ST followed by AT) in blood pressure responses in normotensive individuals and found results that support those observed in the present study. The authors found PEH in SBP at 30 and 45 minutes post exercise in the AT session followed by ST. Similar results were also found in the study of Keese, Farinatti, Pescatello, Cunha, and Monteiro (2012) in which the effect of the intensity of the concurrent training on BP found a significant difference in all the post-exercise intervals (minutes 10 to 120 with 10 minute intervals) in the three sessions tested. Keese, Farinatti, Pescatello, and Monteiro (2011) observed an ABP decrease in all the protocols between minutes 10 and 120, however, the protocol was performed at $65 \%$ of the $\mathrm{VO}_{2}$ peak and $80 \%$ of $1 \mathrm{RM}$, which differed from the present study, that used the working heart rate. Besides that, both studies have found higher PEH effects when the ST and the AT were performed simultaneously

Regarding the DBP behaviour, no significant differences were found. Thus, we can assert that there was no PEH in this variable. A similar result was verified by Saldanha et al. (2016) and Brito, Barata, Filho, and Navarro (2010) after evaluating the effect of a single training session similar to the ones of the present study. In this sense, a possible explanation can be presented due to having used trained and healthy subjects, in which many times small alterations are not observed. On the other hand, Keese et al. (2011) have observed a DBP decrease in their three experimental protocols. This fact may be explained by the differences in the protocols used, such as the number of sets, number of exercises, the intervals between the combined exercises and the BP measurement devices. Therefore, the choice of the training protocol choice might have an important interference in the results found and in the different magnitudes of the PEH effect. Another fact may be explained by the clinical condition of the subjects, considering that normotensive individuals were used, and the most expressive values of pressure reduction occur in hypertensive patients (Halliwill, 2001). In addition, post-exercise values depend on pre-exercise values. In the present study the pre-values were low, so they are not expected to drop post-exercise.

The limitations of the present study include a limited number of participants, which makes it difficult to draw definitive conclusions, the 
normotensive condition of study participants, which may have influenced low blood pressure values prior to the execution of exercise protocols, which may explain the small reductions after the exercise protocols. As well, the lack of a control group made it impossible for us to compare the behaviour of blood pressure after performing the exercise protocols.

\section{CONCLUSION}

The SBP may decrease because of the CT in normotensive men when these individuals undergo intensities of $50 \%$ of 1RM for ST and between $80-90 \%$ of the WHR for AT. However, the training model that alternates ST and AT used in this study was not efficient in generating alterations in the post-exercise DBP pressure values. We suggest that new studies evaluate this protocol in hypertensive individuals in order to confirm their efficiency in SBP decrease and to retest the DBP behaviour in this population.

\section{Acknowledgments:}

Nothing to declare.

\section{Conflict of interests:}

Nothing to declare.

\section{Funding:}

Nothing to declare.

\section{REFERENCES}

Akpolat, T., Erdem, E., \& Aydogdu, T. (2012). Validation of the omron $\mathrm{m} 3$ intellisense (hem7051-e) upper arm blood pressure monitor, for self-measurement, according to the european society of hypertension international protocol revision 2010 in a stage 3-5 chronic kidney disease population. Kidney and Blood Pressure Research, 35(2), 82-88. Doi: 10.1159/000330719

Barreto, C. B., Aguiar, S. S., Palmeira, R., Coelho Junior, H. J., Gargaglione, E. M. L., Oliveira, J. F., ... \& Asano, R. Y. (2015). What is the minimum volume of aerobic physical exercise necessary to elicit postexercise hypotension? Journal of Exercise Physiology online, 18(6), 1-12.

Brito, D. C., Barata, P. F., Filho, M., \& Navarro, A. C. (2010). Efeito de uma única seção de exercício de força a $60 \%$ de $1 \mathrm{rm}$ sobre a pressão arterial de indivíduos treinados do gênero masculino. Revista Brasileira de Prescrição e Fisiologia do Exercício, 21 (4),
$257-262$.

Corso, L. M. L., Macdonald, H. V., Johnson, B. T., Farinatti, P., Livingston, J., Zaleski, A. L., ...\& Pescatello, L. S. (2016). Is concurrent training efficacious antihypertensive therapy? a metaanalysis. Medicine \& Science in Sports \& Exercise, 48(12), 2398-2406. Doi: 10.1249/MSS.0000000000001056

Cunha, F. A. , Santos, L. M. , Massaferri, R. O., Monteiro, T. P. L., \& Farinatti, P. V. T. (2013). Hipotensão pós-exercício induzida por treinamento aeróbio, de força e concorrente: aspectos metodológicos e mecanismos fisiológicos. Revista Hospital Universitário Pedro Ernesto, 12(4), 99-110. Doi:10.12957/rhupe.2013.8717

Garber, C. E., Blissmer, B., Deschenes, M. R., Franklin, B. A., Lamonte, M. J., \& Lee, I. M., American College of Sports Medicine (2011). American college of sports medicine position stand. quantity and quality of exercise for developing and maintaining cardiorespiratory, musculoskeletal, and neuromotor fitness in apparently healthy adults: guidance for prescribing exercise. Medicine $\mathcal{E}$ Science in Sports $\mathcal{E}$ Exercise, 43(7), 1334-1359. Doi: 10.1249/MSS.0b013e318213fefb

Gambassi, B. B., Rodrigues, B., Feriani, D. J., Novais, T. M. G., Oliveira, P. L. L., Sauaia, B. A., Almeida, F. J. F., ... \& Mostarda, C. T. (2016). Influence of Non-Periodized Resistance Training on Blood Pressure in Healthy Elderly Women. Journal of Exercise Physiology online, 19(3), 50-58.

Guedes, D. P., \& Guedes, J. E. R. P. (2006). Manual prático para avaliação em educação física (1 ${ }^{\mathrm{a}} \mathrm{Ed}$.). Barueri, SP: Manole.

Halliwill, J. R. (2001). Mechanisms and clinical implications of post-exercise hypotension in humans. Exercise and Sport Science Review, 29(2), 65-70. Doi: 10.1097/00003677-20010400000005

Keese, F., Farinatti, P., Pescatello, L., Cunha, F., \& Monteiro, W. (2012). Aerobic Exercise Intensity Influences Hypotension Following Concurrent Exercise Sessions. International Journal of Sports Medicine, 33(2), 148-153. Doi: 10.1055/s-00311291321.

Keese, F., Farinatti, P., Pescatello, L., \& Monteiro, W. (2011). A Comparison of the Immediate Effects of Resistance, Aerobic, and Concurrent Exercise on Postexercise Hypotension. Journal of Strength and Conditioning Research, 25(5), 1429-1436. Doi: 10.1519/JSC.0b013e3181d6d968

Mazzoccante, R. P., Sousa, I. R. C. de, Pereira, R. M. dos S., Souza, T. F. de L., Moraes, J. F. V. N. de, Campbell, C. S. G., ... Campbell, C. S. G. (2016). Efeitos da alternância entre exercícios aeróbicos e resistência exercício em diferentes sessões de exercício concorrente em respostas pressão arterial de atletas: um estudo randomizado. Revista Brasileira de Educação Física e Esporte, 30(2), 235-243. Doi: 10.1590/1807- 
38 | ALF Medeiros, MV Sousa, M Lunardi, SN Oliveira, CR Freitas

55092016000200235

Mediano, F. M. F., Paravidino, V., Simão, R., Pontes, F. L., \& Polito, M. D. (2005). Comportamento subagudo da pressão arterial após o treinamento de força em hipertensos controlados. Revista Brasileira de Medicina do Esporte, 11(6), 337-340.

Polito, M. D., \& Farinatti, P. T. V. (2006). Blood pressure behavior after counter-resistance exercises: a systematic review on determining variables and possible mechanisms. Revista Brasileira de Medicina do Esporte, 12(6), 386-392.

Saldanha, M. A., Vilaça-Alves, J., Neto, G. R., Morgado, N. M., Saavedra, F., Lemos, R., Moreira, T. R., ... \& Reis, V. M. (2016). Acute effect of resistance exercise performed at different intensities on the hemodynamics of normotensive men. Motricidade, 12(1), 60-68. Doi: 10.6063/motricidade.6020

Santiago, D. A., Moraes, J. F. V. N., Mazzocante, R. P., Boullosa, D. A., Simões, H. G., \& Campbell, C. S. G. (2013). Corrida em esteira e exercícios de força: efeitos agudos da ordem de realização sobre a hipotensão pós-exercício. Revista Brasileira de Educação Física e Esporte, 27(1), 67-73. Doi: 10.1590/S1807-55092013000100007.

Senna, G., Queiroz De Oliveira, C., Kreuger, S., Scudese, E., Monteiro, W., Boone, T., ... \& Zhou, B. (2016). Hypotensive Effect of Resistance Training Performed on Stable vs. Unstable Surfaces. Journal of Exercise Physiology online, 19(1), 17-26.

Tanaka, H., Monahan, K. D., \& Selos, D. R. (2001). Age-predicted maximal heart rate revisited. Jornal of the American College of Cardiology, 37(1), 153156. Doi: 10.1016/S0735-1097(00)01054-8.

Vilaça-Alves, J., Neto, G. R., Morgado, N. M., Saavedra, F., Lemos, R., Moreira, T. R., ... \& Reis, V. M. (2016). Acute Effect of Resistance Exercises Performed by the Upper and Lower Limbs with Blood Flow Restriction on Hemodynamic Responses. Journal of Exercise Physiology online, 19(3), 100-109.

All content of Journal Motricidade is licensed under Creative Commons, except when otherwise specified and in content retrieved from other bibliographic sources. 